\title{
Recording low spatial frequencies while maintaining information limit resolution using the TEAM I microscope
}

\author{
S.J Haigh*, B. Jiang**, D. Alloyeau***, C. Kisielowski***, A.I.Kirkland* \\ * Department of Materials, University of Oxford, Parks Rd., Oxford, OX1 3PH, UK ** FEI Company, \\ NE Dawson Creek Dr., Hillsboro Oregon, 97124, *** National Center for Electron Microscopy, \\ Lawrence Berkeley National Laboratory, One Cyclotron Rd., Berkeley CA 94720
}

A next generation electron microscope has been developed within DoE's TEAM Project [1]. The TEAM I microscope is the final product of this effort and makes available a post specimen chromatic aberration corrector (Cc) [2] to provide deep sub $\AA$ resolution. Operation voltages currently range from $80 \mathrm{kV}$ to 300 $\mathrm{kV}$. In its standard broad beam mode at $300 \mathrm{kV}$, the instrument allows for the transmission of high spatial frequencies up to a limit of $19 \mathrm{~nm} 1(0.05 \mathrm{~nm})$. However, low spatial frequencies are absent from high resolution images due to the intrinsic properties of the contrast transfer function (CTF). Although this is not usually a concern for small unit cell structures, many materials systems have characteristic sizes that exceed $1 \mathrm{~nm}$. In order to enable the phase contrast imaging of large objects, it is common practice to record images at high defocus, thereby changing the CTF so that low spatial frequencies are transmitted. The compromise is that this only occurs at the expense of transfer of high resolution information which is not transferred. This drawback has driven the development of physical phase plates for TEM [3]. In this contribution we report on an alternative way to capture low spatial frequencies while maintaining information transfer to high spatial frequencies using a Cc corrected instrument and exit wave reconstruction. To demonstrate this approach we have utilized the TEAM 1 microscope operated in imaging mode with a low beam convergence $(\sim 20 \mathrm{rad})$ together with a $\mathrm{Cc} / \mathrm{Cs}$ corrector. Figure 1 demonstrates that if $\mathrm{Cc}$ and $\mathrm{Cs}$ are chosen to be close to zero information transfer to about $0.1 \mathrm{~nm}$ can be maintained even at low accelerating voltages and at large underfocus values. It is also seen from Figure 1 that for a phase object imaged at an underfocus of $2 \mathrm{~m}$, low resolution information is transferred at $17 \mathrm{~nm}$. The rapid CTF oscillations introduced at large underfocus values cause unavoidable image delocalization as shown in figure 2. However, this can be eliminated by reconstructing the electron exit wave function for a focal series of images over a very large focal range. Thus, this approach mimics the effect of a phase plate. In Figure 2 we demonstrate the applicability of this concept by reconstructing the exit wave phase of a multiwall carbon nanotube decorated with molecules. The data set used for reconstruction consisted of a series of 80 images with a nonuniform focal step and a focal range between $0.8 \mathrm{~m}$ and $10 \mathrm{~nm}$. The reconstruction recovers information in range from $\sim 5 \mathrm{~nm}$ to below $0.2 \mathrm{~nm}$ although some image blur occurs probably due to a beam induced motion of the molecules [4].

References

1. U. Dahmen, R. Erni, V. Radmilovic, C. Kisielowski, et al., Phil. Trans. R. Soc. A 367, (2009) 37953808

2. B. Kabius, P. Hartel, M. Haider, H. Müller, et al., J. Electron Micro. (2009) 1-9

3. D. Alloyeau, W.K. Hsieh, E.H. Anderson, D.L. Hilken, X. Meng, et. al., Ultramicroscopy (2010), in press

4. The TEAM project is supported by the Department of Energy, Office of Science, Basic Energy Sciences. Financial support from EPSRC grant number EP/F048009/1 is acknowledged 


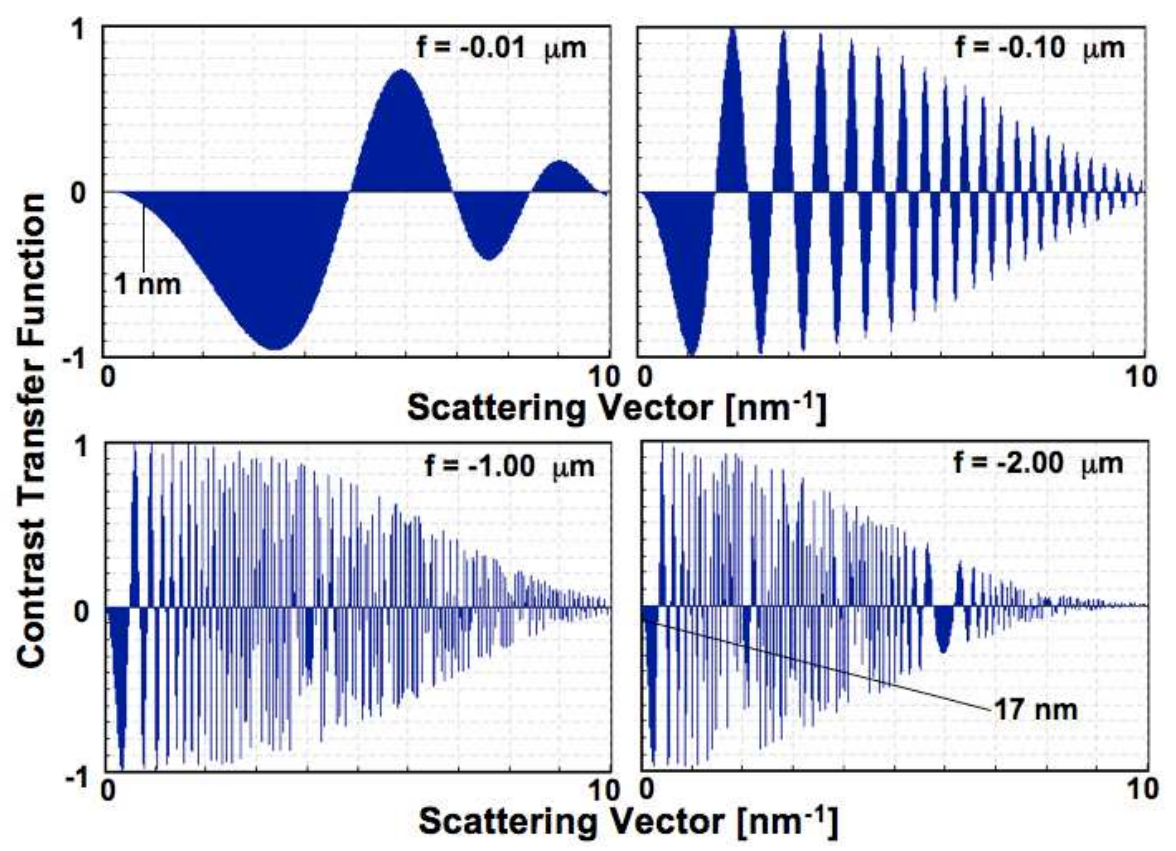

FIG. 1. Contrast transfer functions for $\mathrm{Cc}=\mathrm{Cs}=0$, beam divergence: $20 \mu \mathrm{rad}$, acceleration voltage $=$ $80 \mathrm{kV}$, Focus spread: $2 \mathrm{~nm}$, underfocus values as indicated.

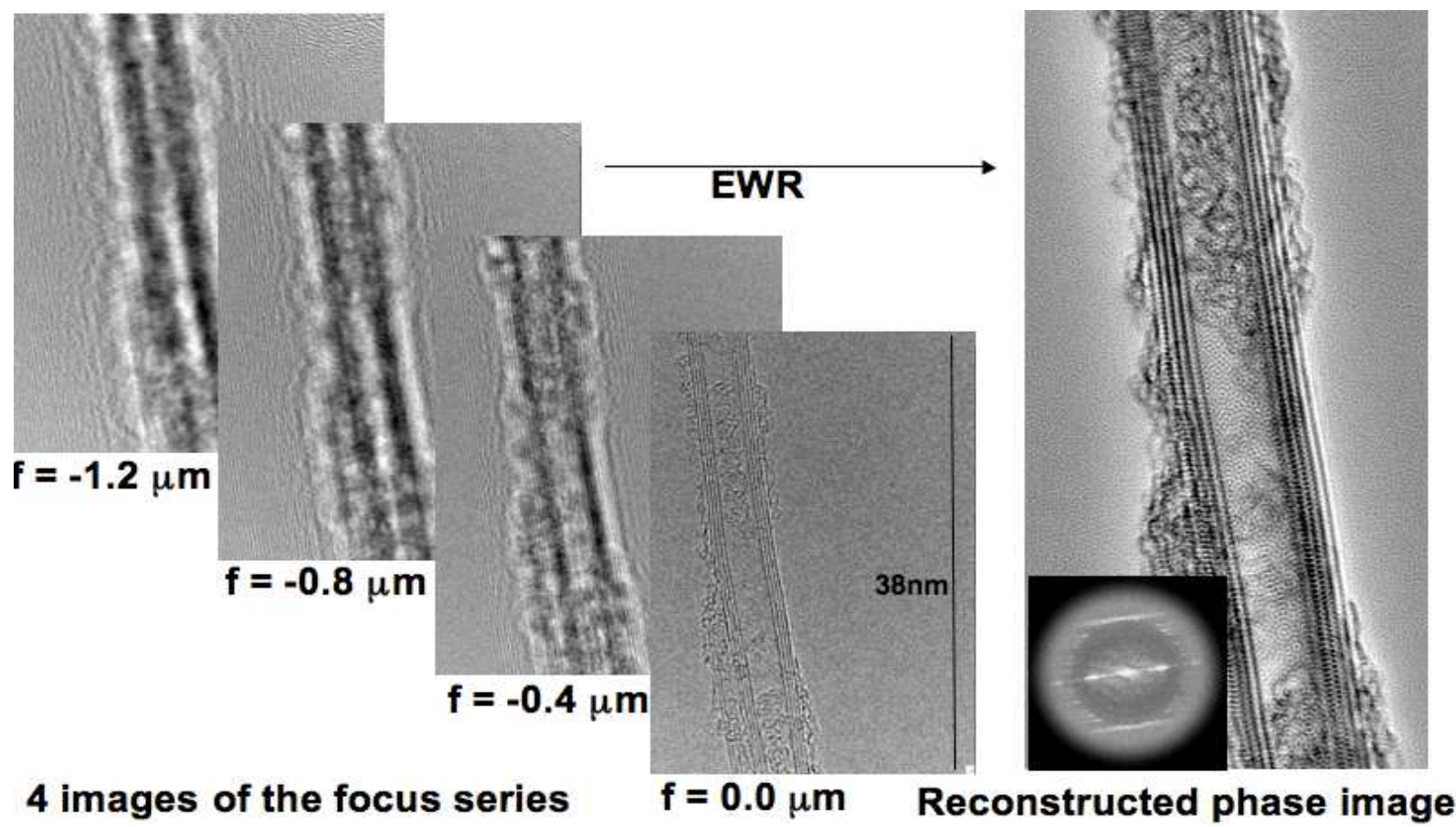

FIG. 2. Multi-wall carbon nanotubes decorated with molecules imaged with different focus values as indicated. Note the large underfocus values. Transmission of all spatial frequencies from $\sim 5 \mathrm{~nm}$ to below $0.2 \mathrm{~nm}$ was achieved by reconstructing the phase of the electron exit wave. 